\title{
Local decomposition of hybridization functions: chemical insight into correlated molecular adsorbates
}

\author{
Marc Philipp Bahlke ${ }^{*}$, Michaela Schneeberger ${ }^{1}$, and Carmen Herrmann ${ }^{1^{*}}$ \\ ${ }^{1}$ University of Hamburg, Department of Chemistry, HarBor Bldg. 610, \\ Luruper Chaussee 149, 22761 Hamburg, Germany \\ *Corresponding authors: marc.philipp.bahlke@chemie.uni-hamburg.de, \\ carmen.herrmann@chemie.uni-hamburg.de
}

January 27th 2020

\begin{abstract}
Hybridization functions are an established tool for investigating the coupling between a correlated subsystem (often a single transition metal atom) and its uncorrelated environment (the substrate and any ligands present). The hybridization function can provide valuable insight into why and how strong correlation features such as the Kondo effect can be chemically controlled in certain molecular adsorbates. To deepen this insight, we introduce a local decomposition of the hybridization function, based on a truncated cluster approach, enabling us to study individual effects on this function coming from specific parts of the systems (e.g., the surface, ligands, or parts of larger ligands). It is shown that a truncated-cluster approach can reproduce the Co $3 d$ and Mn $3 d$ hybridization functions from periodic boundary conditions in $\mathrm{Co}(\mathrm{CO})_{4} / \mathrm{Cu}(001)$ and $\mathrm{MnPc} / \mathrm{Ag}(001)$ qualitatively well. By locally decomposing the
\end{abstract}


hybridization functions, it is demonstrated at which energies the transition metal atoms are mainly hybridized with the substrate or with the ligand. For the Kondo-active the $3 d_{x^{2}-y^{2}}$ orbital in $\mathrm{Co}(\mathrm{CO})_{4} / \mathrm{Cu}(001)$, the hybridization function at the Fermi energy is substrate-dominated, so we can assign its enhancement compared with ligand-free Co to an indirect effect of ligand-substrate interactions. In $\mathrm{MnPc} / \mathrm{Ag}(001)$, the same is true for the Kondo-active orbital, but for two other orbitals, there are both direct and indirect effects of the ligand, together resulting in such strong screening that their potential Kondo activity is suppressed. A local decomposition of hybridization functions could also be useful in other areas, such as analyzing the electrode self-energies in molecular junctions. 


\section{Introduction}

When spin-polarized atoms or molecules are brought in contact with a metallic substrate, their interaction with the metal's conduction band electrons can lead to a screening of their unpaired electron(s) below a certain temperature [1-11]. This is a realization of strong correlation known as the Kondo effect, observable in scanning tunneling spectra as an anomal behaviour at zero bias voltage [12-14]. The Kondo effect can act as evidence for spin polarization on adsorbates [15-17]. Furthermore, being able to control the Kondo effect chemically or mechanically [18 26] might be interesting for spintronic applications such as tailoring electronic and magnetic properties at organic-semiconductor-metal interfaces [27].

The Kondo effect is a complex, not yet entirely understood phenomenon (it has even been suggested very recently that the signatures assigned to the Kondo effect in "classic" examples of atomic adsorbates may in fact result from magnetic anisotropy [28]). Accurate theoretical models that include an adequate description of electron correlation in Kondo systems [29] are often very costly $\left.\right|^{1}$

A qualitative picture may be drawn by using less expensive theoretical approaches, e.g., by analyzing the energy-dependent hybridization function based on a Kohn-Sham density functional theory (KS-DFT) calculation. The hybridization function is related to the density of states of an environment which is "seen" by the Kondo-relevant orbitals of the adsorbate. Simple models suggest that the imaginary part of the hybridization function close to the Fermi energy plays a major role for the magnitude of the Kondo effect [33]. For understanding and controlling the Kondo effect, it would therefore be desirable to quantify how the hybridization function is affected by different parts of the molecule-adsorbate system. For example, if the unpaired spin is located on a transition metal atom surrounded by one or several ligands, to what extent is the hybridization function determined by interactions of the unpaired spin with the ligands, and to what extent by interactions with the surface? So

\footnotetext{
${ }^{1}$ Cost-effective approaches to certain classes of strongly correlated systems are currently being developed based on machine learning [30]. Also note that the relatively cheap DFT can, in principle, describe correlation in spin-polarized systems exactly, even though in practice this is often not the case with today's approximate exchange-correlation functionals [31, 32].
} 
far, such questions have been adressed by removing different parts of the system (e.g the ligands) and by comparing the resulting hybridization function with the full one [34, 35]. This has the drawback that removing parts of the system may affect its electronic structure in undesired ways.

We therefore propose a local decomposition of hybridization functions, motivated by chemical concepts such as partial charges [36-47]. This allows for analyzing contributions coming from, e.g., ligands or single atoms to the hybridization function directly, without having to cut away parts of the structures. For its ease of access to well-defined local basis functions, we will work with KS-DFT calculations on truncated systems employing atom-centered basis sets, i.e., with clusters modeling the extended metal surfaces. Therefore, an additional question will be whether such clusters (of a reasonably large size) can produce hybridization functions comparable to those obtained from the more standard approach under periodic boundary conditions combined with a plane-wane basis.

After first introducing the role of hybridization functions in the Anderson impurity model of Kondo systems (Section 2) and then their local decomposition (Section 3), we will describe an implementation of this decomposition (Section 4) and then illustrate its capacities by applying it to two examples from the literature (Section 5)

\section{Hybridization Functions in the Anderson Impurity Model}

The Anderson impurity model (AIM) has become well established for studying materials with strong electron correlation on a defined set of (usually local) orbitals, often referred to as the correlated subspace or correlated subsystem. This (local) correlation accounts for only a small part of the total energy, but it can lead to interesting behaviors observable in different kind of experiments such as the zero-bias anomaly in scanning tunneling spectroscopy, caused by the Kondo effect. 
The term "impurity" refers to a magnetic adsorbate on a (metal) surface, often a transition metal atom with or without surrounding ligands. The correlated subspace is then usually defined as the $d$ orbitals of that transition metal atom (or a subset of them, depending on how they interact with the substrate and among themselves) $)^{2}$. For a transition metal complex adsorbed on a substrate, the environment would include both the ligands and the substrate.

For multiple orbitals in the correlated subspace, the general form of the Anderson Hamiltonian reads,

$$
\hat{H}=\sum_{\nu \sigma} \epsilon_{\nu} \hat{c}_{\nu, \sigma}^{\dagger} \hat{c}_{\nu, \sigma}+\sum_{\nu i \sigma}\left[V_{\nu i} \hat{c}_{\nu, \sigma}^{\dagger} \hat{d}_{i, \sigma}+V_{\nu i}^{*} \hat{d}_{i, \sigma}^{\dagger} \hat{c}_{\nu, \sigma}\right]+\sum_{i \sigma} \epsilon_{i} \hat{d}_{i \sigma}^{\dagger} \hat{d}_{i \sigma}+\frac{1}{2} \sum_{\substack{i j k l \\ \sigma \sigma^{\prime}}} U_{i j k l} \hat{d}_{i \sigma}^{\dagger} \hat{d}_{j \sigma^{\prime}}^{\dagger} \hat{d}_{l \sigma^{\prime}} \hat{d}_{k \sigma}
$$

$\epsilon_{i}$ is the effective single-particle energy of a local orbital $i$ of the impurity, with the corresponding creation and annihilation operators for electrons with spin $\sigma, \hat{d}_{i \sigma}$ and $\hat{d}_{i \sigma}^{\dagger} ; \epsilon_{\nu}$ is the energy of an environment orbital $\nu$ (the environment is often called the electronic bath, see Figure 1] with the corresponding creation and annihilation operators $\hat{c}_{\nu \sigma}$ and $\hat{c}_{\nu \sigma}^{\dagger}$. The electrons of the environment are electronically coupled to the correlated subsystem via the hybridization parameters $V_{\nu i}$, and $U_{i j k l}$ is the local Coulomb interaction within the correlated subspace as introduced by Slater [52].

For details on explicitly solving the AIM we refer the reader to References [33, [53, 54]. Here, we just point out that the AIM is often parametrized by an effective single-particle wave function method (e.g. Kohn-Sham density functional theory, resulting in so-called DFT ++ approaches). When solving the AIM with, e.g., Monte Carlo methods, the relevant information on the environment and its coupling to the correlated subsystem is encoded in the energy-dependent hybridization function $\Delta_{i j}(\omega)$ [53, 55],

\footnotetext{
${ }^{2}$ Which orbitals are relevant for understanding Kondo physics can be a matter of debate [29, 48, 49]. Also, progress towards describing the Kondo effect in organic radical adsorbates is being made, even though such systems still pose a challenge in this context due to their delocalized orbitals [50, 51]
} 


$$
\Delta_{i j}(\omega)=\lim _{\eta \rightarrow 0+} \sum_{\nu} \frac{V_{\nu i} V_{\nu j}^{*}}{\omega+i \eta-\epsilon_{\nu}}
$$

Here, in is an infinitesimally small offset (or smearing parameter), and $\omega$ is the (effective single-particle) energy. In practice, $\eta$ is set to a small finite value, and the smaller this value, the sharper the features in the energy-dependent hybridization function will be.

$\lim _{\eta \rightarrow 0+}\left(\omega+i \eta-\epsilon_{\nu}\right)^{-1}$ is the Green's function associated with an environment orbital. The imaginary part of a Green's function is related to the density of states, and accordingly, $\operatorname{Im}\left[\Delta_{i j}(\omega)\right]$ can be thought of as a density-of-states for the environment, where each "state" (or rather environment orbital) is weighted by its (squared) coupling to the correlated subsystem. This hybridization function is equivalent to what is called the self-energy for subsystems embedded in an environment in other contexts, for example in single-molecule conductance [56, 57].

The interaction of a correlated-subsystem orbital with the environment leads to an energy broadening of this orbital via the imaginary part of the energy-dependent hybridization function, and to an energy shift via the real part of that function. For understanding chemical structure-property relationships governing the Kondo effect, it would be interesting to know which parts of the environment cause such broadening and shifting. This is particularly interesting when the environment consists of both ligands and a substrate, and it could allow analyzing the effect of chemical modifications, such as ligand substitution or removal, on the hybridization function and, consequently, on the Kondo properties. In principle, the importance of different parts of the environment can be assessed by cutting away those parts. This was done in Ref. [35]. However, such a procedure does not take into account that different parts of the environment do not only influence the correlated subsystem, but also each other electronically. Therefore, we will introduce a local decomposition of the energy-dependent hybridization function in the following. 


\section{Introducing a local decomposition of hybridization functions}

In many electronic-structure calculations on finite (and also on some periodic) systems, the spatial parts of the molelcular orbitals are expanded in a basis of $n$ non-orthogonal atomcentered functions $\{|a\rangle\}$,

$$
\phi_{i}=\sum_{a=1}^{n} C_{a i}|a\rangle
$$

with generally non-zero overlap between different basis functions. We transform our nonorthogonal basis $\{|a\rangle\}$ into a Löwdin-orthogonalized basis $\left\{\left|a^{\prime}\right\rangle\right\}$ [36, 58,

$$
\left|a^{\prime}\right\rangle=\sum_{b=1}^{n}\left(\mathbf{S}^{-1 / 2}\right)_{a b}|b\rangle
$$

so that we have $S_{a b}^{\prime}=\left\langle a^{\prime} \mid b^{\prime}\right\rangle=\delta_{a b}$. A Löwdin-orthogonalized basis is in not strictly atomcentered, which is why our partial hybridization functions introduced below are not entirely locally defined. However, it remains sufficiently local to be applied successfully for, for example, population analysis of a broad range of system in quantum chemistry [59]. We also minimize the delocalization problem here by working in a relatively small atom-centered basis set (see Methodology section).

We now want to calculate the hybridization function of the subsystem $C$ spanned by the basis functions $\left\{\left|i^{\prime}\right\rangle\right\}$. The subsystem $C$ represents in our case the impurity orbitals, which, in the context of strongly correlated materials, are usually the $d$ or $f$ orbitals of an atom (see Figure 1). Consequently, the remainder of our system represents the electronic bath $B$. In general, the subsystem $C$ could also be extended to include other orbitals, such as $s$ and $p$ orbitals or delocalized molecular orbitals (MOs), and it is thus not only restricted to a single atom (see for instance References [50, 51]). 


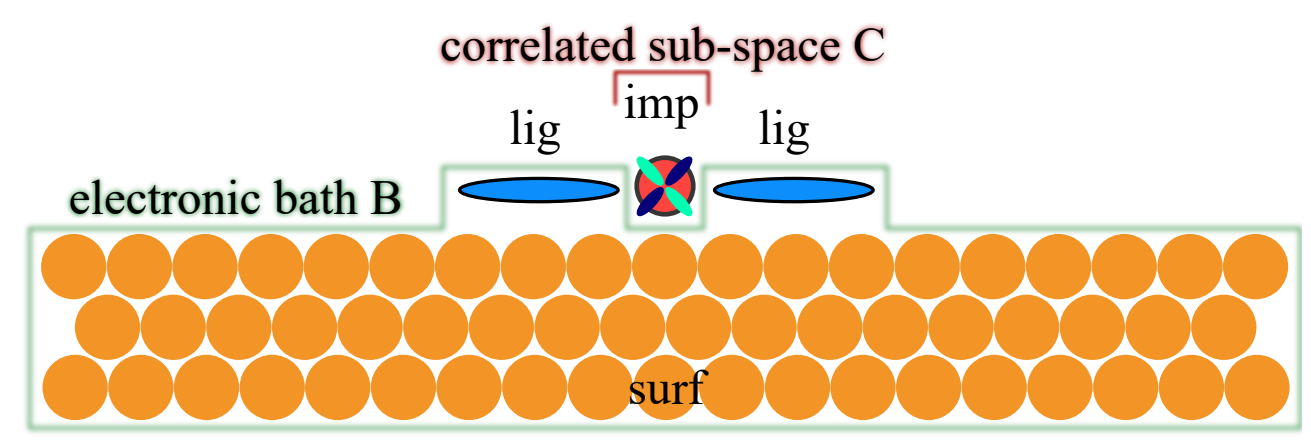

Figure 1: Schematic illustration of a molecule on a surface (surf), with one impurity (imp) atom and two ligands (lig). The correlated subsystem $C$ contains selected basis functions of the impurity atom (in this work, the $3 d$ orbitals of a transition metal atom). Note that $C$ can in general be extended to more orbitals, including those on different kinds of atoms, as e.g. in Reference [50]. The electronic bath $B$ contains all basis functions of the ligands, the surface, and the remaining basis functions of the impurity atom (e.g., $s^{-}, p^{-}$, or diffuse and polarization functions).

We start with transforming the effective single-particle Hamiltonian matrix $\mathbf{H}$ into the Löwdin-orthogonalized basis,

$$
\mathbf{H}^{\prime}=\mathbf{S}^{-1 / 2} \mathbf{H S}^{-1 / 2} \text {. }
$$

To evaluate the hybridization function, it is required to find a representation of $\mathbf{H}^{\prime}$ in which the electronic bath $B$ and the correlated sub-system $C$ are diagonal within their individual blocks. This can be achieved by finding a suitable transformation matrix $\mathbf{T}$ that fullfills this requirement (see also Figure 2),

$$
\overline{\mathbf{H}}=\mathbf{T}^{\dagger} \mathbf{H}^{\prime} \mathbf{T} .
$$

Here, $\mathbf{T}$ is a diagonalization matrix of dimension $n \times n$, which contains the eigenvectors of the bath and correlated subspace Hamiltonians in its diagonal blocks $\mathbf{T}_{\mathrm{C}}$ and $\mathbf{T}_{\mathrm{B}}$, while the entries in the off-diagonal blocks are zero. This overall procedure of block-diagonalizing is similar to approaches employed for evaluating charge transfer dynamics between molecules 
and surfaces [60, 61]. In this context, an improved scheme combining orthogonality with localization has been recently suggested [62].
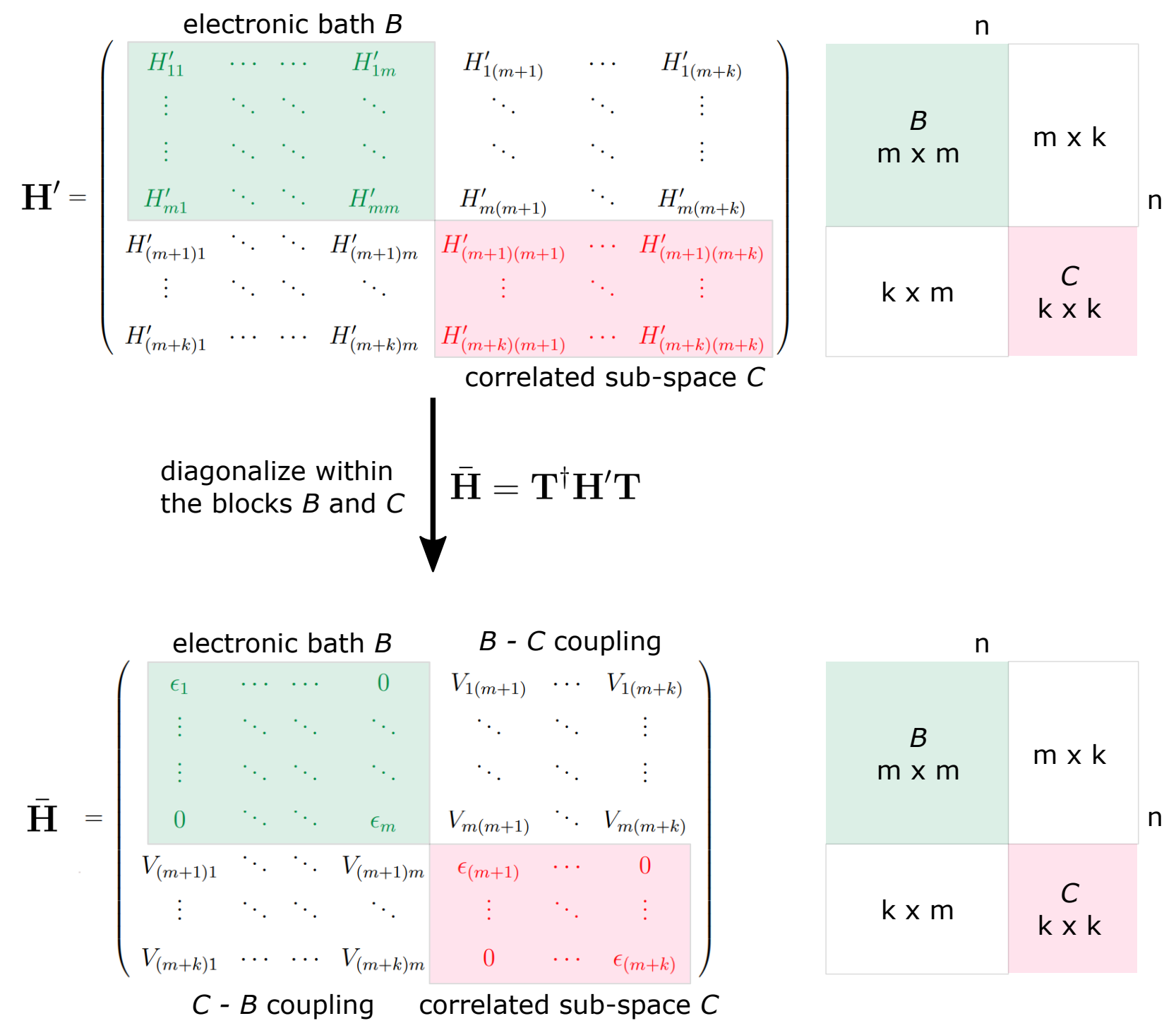

Figure 2: Depiction of the transformation described by Equation (6). The Löwdinorthogonalized single-particle Hamiltonian matrix $\mathbf{H}^{\prime}$ is diagonal within the electronic bath and correlated subsystem blocks.

In the following, we introduce a scheme to decompose the hybridization function $\bar{\Delta}_{i j}(\omega)$ (evaluated in the block-diagonal basis illustrated in Figure 2 and simply written $\Delta_{i j}(\omega)$ from here on) into different parts, so that we can quantify the contribution of different parts of the electronic bath to the full hybridization function. A possible choice would be the 
decomposition into a ligand and a surface part, as depicted in Figure 1. In the case of more than one ligand, it is also possible to analyze the contribution of each ligand individually, which is promising for studying their individual effects on the hybridization function of the correlated subsystem (the impurity orbitals).

For this reason, we weight the sum terms making up the hybridization function by $\sum_{a} c_{\nu a}^{2}$, where the coefficients $c_{\nu a}$ are those of the bath MOs $|\nu\rangle$ appearing in the columns of the diagonalization matrix $\mathbf{T}_{\mathrm{B}}$. This sum is 1 if the sum runs over all elements of space $B$ (the electronic bath) and if the bath orbitals are normalized,

$$
\sum_{\text {part }} \sum_{a \in \text { part }} c_{\nu a}^{2}=1
$$

Truncating the sum over $a$ to basis functions of a specific part of the system yields a local or partial hybridization function,

$$
\Delta_{i j}^{\mathrm{part}}(\omega)=\lim _{\eta \rightarrow 0+} \sum_{\nu} \sum_{a \in \mathrm{part}} \frac{c_{\nu a}^{2} V_{\nu i} V_{\nu j}^{*}}{\omega+i \eta-\epsilon_{\nu}}
$$

where "part" can contain the basis functions of the ligands or the surface (or in principle any part of the electronic bath). Following our example of Figure 1, the full hybridization function can be partitioned as

$$
\Delta_{i j}(\omega)=\Delta_{i j}^{\text {ligand }}(\omega)+\Delta_{i j}^{\text {surface }}(\omega)+\Delta_{i j}^{\text {remainder }}(\omega)
$$

where $\Delta_{i j}^{\text {remainder }}(\omega)$ refers to the remaining basis functions of the impurity atom (i.e., all basis functions centered on the impurity atom that do not make up the $3 d$ shell, such as the $s$ and $p$ shells or polarization functions, or also additional $3 d$ functions in case of double or triple zeta basis sets). These remainder contributions are small in the examples studied below, so we will not discuss them in the following.

For a deeper analysis of how molecules can interact with surfaces, one may also wish to 
evaluate the hybridization function (and its decomposition) for orbitals which are not part of the correlated subsystem (e.g., in one of our examples below, for the carbon $2 p$ - orbitals of the ligand).

In principle, both the diagonal and the off-diagonal terms of the energy-dependent hybridization function $\Delta_{i j}$ can be important when solving the AIM. In cubic symmetry and in the absence of spin-orbit coupling, the off-diagonal terms are zero [63], and also in the general case, so far often only the diagonal terms of the have been taken into account when solving the AIM, with the off-diagonal ones being neglected ${ }^{3}$. Analogously, the off-diagonal terms have so far mostly been analyzed in special cases such as coupled impurities with competing Kondo and Ruderman-Kittel-Kasuya-Yosida (RKKY) interactions [66] or when spin-orbit coupling plays a role [65]. Otherwise, in-depth discussions of the hybridization function have almost exclusively focused on its diagonal terms. For our local decomposition analysis, we will therefore focus on the diagonal elements of the energy-dependent hybridization function.

Within a simple model describing a single impurity orbital coupled to a bath, the highest temperature at which the Kondo effect can be observed (the Kondo temperature $T_{\mathrm{K}}$ ) is related to the imaginary part of the hybridization function at the Fermi energy [33],

$$
k_{\mathrm{B}} T_{\mathrm{K}}=\frac{\sqrt{-\operatorname{Im} \Delta_{i i}\left(E_{F}\right) U}}{2} \exp \left(\frac{\pi \epsilon_{i}\left(\epsilon_{i}+U\right)}{-\operatorname{Im} \Delta_{i i}\left(E_{F}\right) U}\right) \text {, }
$$

where $k_{\mathrm{B}}$ is the Boltzmann constant, and $U$ the Coulomb interaction on the impurity orbital, i.e., the energy penalty for adding a second electron (of opposite spin) to the orbital, and $\epsilon_{i}$ is the energy of the impurity orbital relative to $E_{F}$. Therefore, we will focus on the imaginary part of the hybridization function when performing our local decomposition in the following.

\footnotetext{
${ }^{3}$ For example, the well-established electronic structure program package ABINIT has introduced the option of nonzero off-diagonal elements in the hybridization function into its density-density continuous-time quantum Monte Carlo (QMC) module just in its very recent version 9.2 in September 2020 [64]. Also, the off-diagonal terms may be related to sign problems in the QMC [65]
} 


\section{Hydra: Hybridization Decomposition — a Rapid Analysis tool}

HYDRA is a computer program to evaluate the hybridization function $\boldsymbol{\Delta}(\omega)$ and its local decompositions $\boldsymbol{\Delta}^{\mathrm{part}}(\omega)$, as shown in Section 3, from DFT output. Additionally, HYDRA can compute the hybridization function using a local Green's functions approach which can be used to evaluate the total density of states (see Section 3 in the supplementary material). An overview of HYDRA's program structure is given in Fig. 3 .

HydRA was implemented in $\mathrm{C}++$ using the EIGEN [67] library to optimize matrix operations. To calculate $\boldsymbol{\Delta}(\omega)$ and a local hybridization $\boldsymbol{\Delta}^{\text {part }}(\omega)$ in the "direct" way as defined in Eq. (8), the overlap matrix of an atom-centered basis set $\mathbf{S}$, the Kohn-Sham (KS) Hamiltonian matrix $\mathbf{H}$, the KS eigenvalues arranged on the diagonal of a matrix $\boldsymbol{\epsilon}$, and the MO coefficients C of a converged density functional theory (DFT) calculation are required. Additionally, when computing $\boldsymbol{\Delta}^{\text {part }}(\omega)$, a list of the basis functions contained in the environment part of interest ("part") are required as input. Based on user input, the correlated subsystem and the bath blocks of $\mathbf{H}$ are diagonalized (the correlated subspace may also be already diagonal, e.g. when it consists of a set of $3 d$ orbitals), and $\boldsymbol{\Delta}^{\text {part }}(\omega)$ and $\boldsymbol{\Delta}(\omega)$ are evaluated. Alternatively, $\boldsymbol{\Delta}(\omega)$ is computed using a Green's function approach, for which a hybrid parallelization using OPENMP [68] and MPI [69] was implemented to decrease computation time (see the supplementary material).

\section{A local analysis of hybridization functions}

It can be advantageous to truncate a surface for studying the properties of adsorbates [7076], or for simulating the electron transport properties through molecules in a molecularjunction setup [77 81]. This is called a (truncated) cluster approach in this work. One major reason for this is the ease of employing a wide range of approximate exchange-correlation 


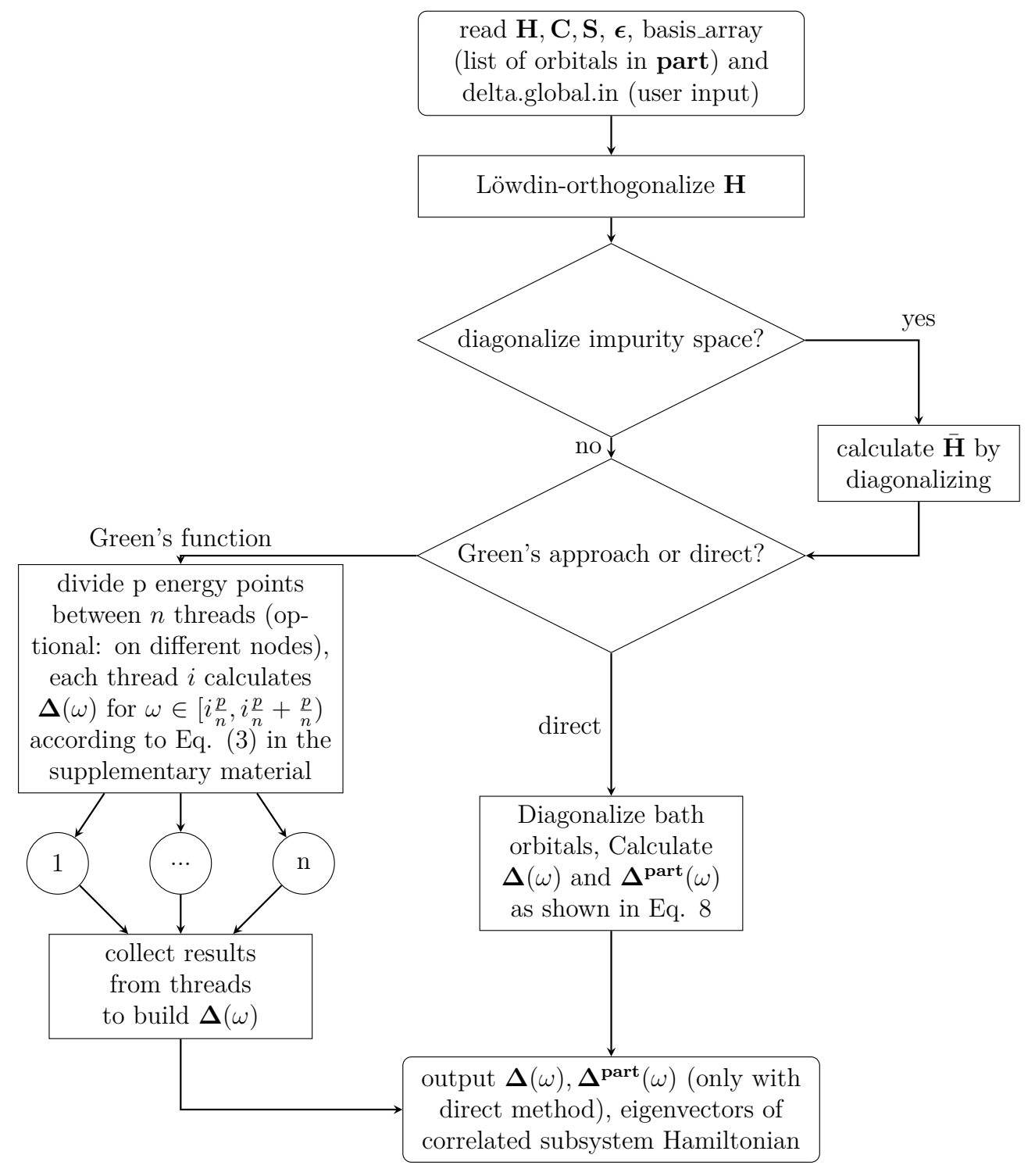

Figure 3: Flowchart for the evaluation of the hybridization function by the the "direct" way as defined in Eq. (8) or by a Green's function approach, and its local decomposition. When diagonalization of the impurity orbitals is chosen, the orbitals might not appear in the original order (e.g., $\mathrm{d}_{\mathrm{z}^{2}}, \mathrm{~d}_{\mathrm{xz}}, \mathrm{d}_{\mathrm{yz}}, \mathrm{d}_{\mathrm{xy}}, \mathrm{d}_{\mathrm{x}^{2}-\mathrm{y}^{2}}$ for $3 d$ orbitals from TuRBOMOLE output). For this reason, we print out the matrix used to diagonalize the correlated subsystem (which contains the eigenvectors of the correlated-subsystem Hamiltonian matrix). The highest absolute value of each column denotes which orbital contributed most to the resulting impurity MO, and considering this, one can assign the correct orbital to the output. 
functionals available in electronic structure programs targeted at finite structures, and the frequently used atom-centered basis functions in such codes (instead of the plane-wave basis common in many periodic boundary conditions (PBC) solid-state codes), which can be beneficial for obtaining chemical insight into these systems $4^{4}$ such as population analysis or bond orbital analysis [84]. In particular, as discussed in Section 3, using an atom-centered basis set makes it possible to divide the hybridization function of a correlated sub-shell into different contributions from individual ligands or the surface (Equation (8)). Such a local decomposition of hybridization functions may provide insights into structure-property relations for Kondo systems.

We employ this combination of a local decomposition of the hybridization function and a cluster approach to study for $\mathrm{Co}(\mathrm{CO})_{4} / \mathrm{Cu}(001)$ how the $\mathrm{CO}$ ligands affect the $\mathrm{Co} 3 d$ hybridization function around the Fermi energy. For this system, it was found experimentally that increasing the number of CO ligands increases the Kondo temperature [85, 86]. As a second example, we will briefly focus on manganese phthalocyanine ( $\mathrm{MnPc}$ ) adsorbed on $\mathrm{Ag}(001)$. For this system, it was recently reported that only one of the five magnetic orbitals contributes to an experimentally observed Kondo effect [87]. The remaining orbitals have either no hybridization at the Fermi energy or are possibly too strongly coupled to one of the ligand orbitals that lies close at the Fermi energy. In both cases, the Kondo activity of the individual orbitals can be rationalized by comparing their hybridization functions with those of the pure $3 d$ atom adsorbates (usually an increase). We will ask in each case how much of this increase comes directly from the ligands, and how much from an indirect mechanism in which the ligands affect the electronic structure of the metal substrate (see Figure 4 .

\footnotetext{
${ }^{4}$ When working with a plane-wave basis, maximally localized Wannier orbitals can also act as a local basis, but this requires a transformation of the MOs as an extra and not necessarily trivial [82, 83] step, and it is not guaranteed that the resulting orbitals are sufficiently localized on the subsystems of interest.
} 


\section{Ligand effects on $\Delta$}

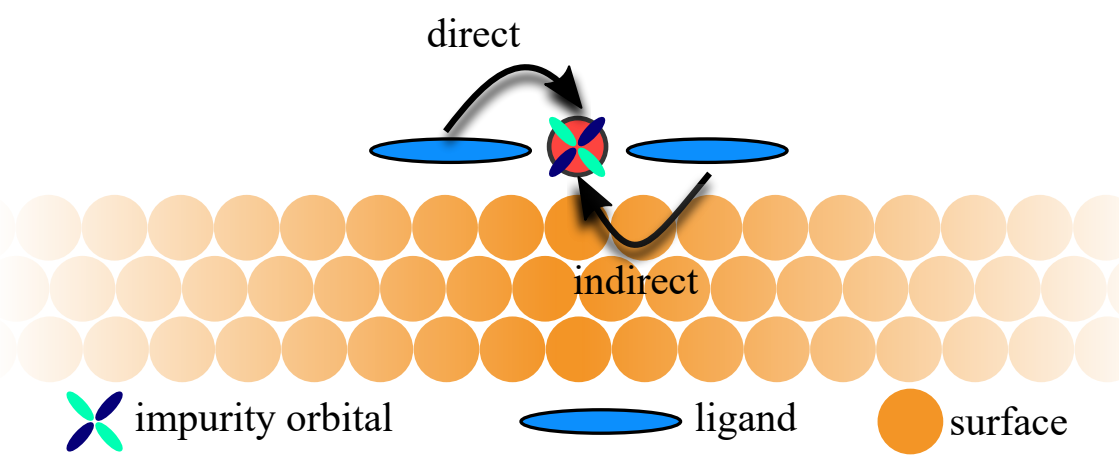

Figure 4: Ligand effects on the impurity hybridization functions via direct ligand-impurity interaction and indirectly via ligand--surface interaction.

\subsection{Methodology}

$\mathrm{Co}(\mathrm{CO})_{4} / \mathrm{Cu}(\mathbf{0 0 1})$ : The $\mathrm{Co}(\mathrm{CO})_{4} / \mathrm{Cu}(001)$ cluster was build from a $C_{4 \mathrm{v}}$-symmetric structure optimized by BLYP-D3 $+U$ under periodic boundary conditions, as discussed in detail in Reference [35]. For modeling the $\mathrm{Cu}(001)$ surface, a pyramid-like cluster with a surface of $6 \times 6 \mathrm{Cu}$ atoms and 6 layers deep, which results in $91 \mathrm{Cu}$ atoms in total (see Figure 5 a)), was cut out from the PBC-optimized $\mathrm{Co}(\mathrm{CO})_{4} / \mathrm{Cu}(001)$ structure. The odd number of $\mathrm{Cu}$ atoms was chosen to enforce an overall closed-shell situation consistent with the DFT parametrization of the Anderson impurity model (AIM) as commonly done in the literature for non-magnetic substrates (the magnetic properties are then taken into account via the subsequent solution of the AIM — even though such a closed-shell treatment of a spinpolarized adsorbate may appear uncommon from a (quantum chemistry) DFT perspective, we have chosen to stick with it for consistency with the DFT ++ literature). 
a)
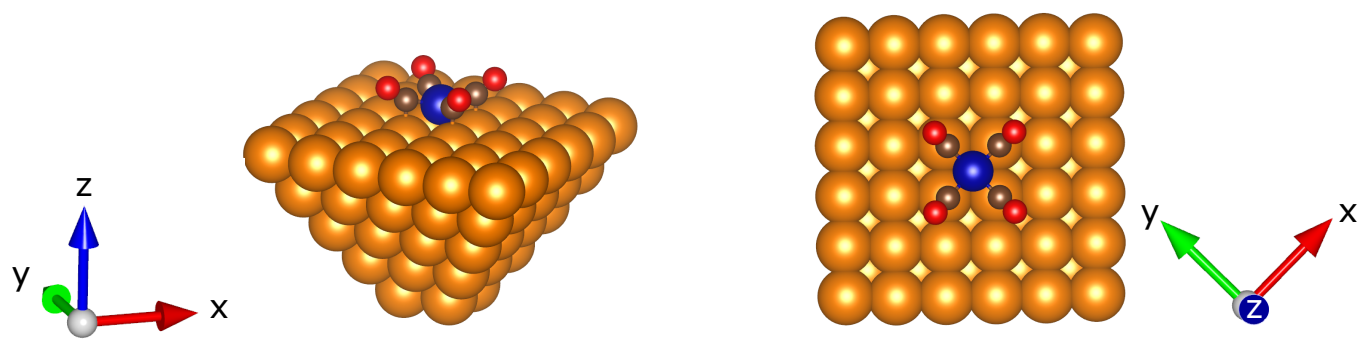

b)
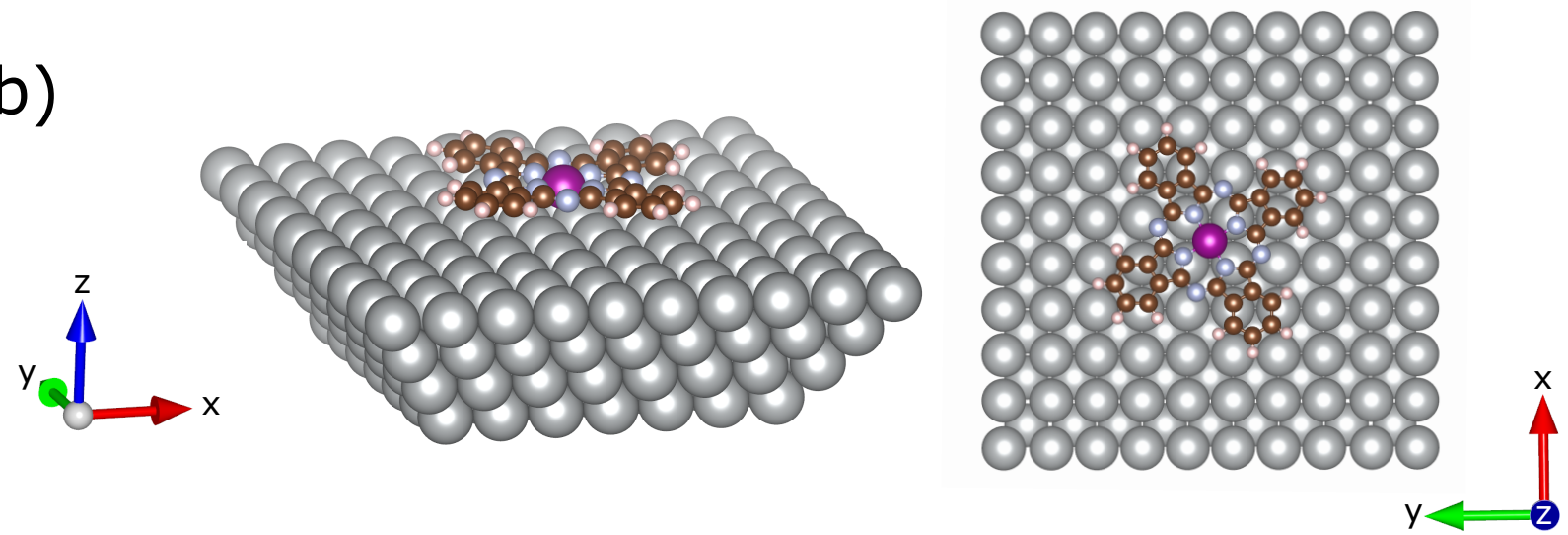

Figure 5: a) $\mathrm{Co}(\mathrm{CO})_{4}$ on a $\mathrm{Cu}_{91}(001)$ cluster. The structure is based on a BLYP-D3+U optimization using periodic boundary conditions, see Reference [35] for details. b) MnPC on a $\mathrm{Ag}_{293}(001)$ cluster. The structure is based on a PBE-D3 optimized MnPC/Ag(001) using periodic boundary conditions (see Methodology for more details).

Based on this structure, DFT single-point calculations were performed with the TURBOMOLE 7.1 program package [88], employing the PBE exchange-correlation functional [89, 90]. Since the clusters are quite large, and to keep the atom-centered single-particle basis as local as possible, a def2-SVP basis set [91, 92]) was employed in the truncated-cluster calculations. The convergence criterion for the energy in the self-consistent-field algorithm was set to $1 \cdot 10^{-6}$ Hartree, and the hybridization functions were evaluated as described in Section 3. To avoid problems with negative gaps between the highest and lowest occupied molecular orbtials (see supplementary material) we applied a thermal (Fermi) smearing with $T=300 \mathrm{~K}$ during the self-consistent-field calculation.

MnPc/Ag(001): To build the $\mathrm{MnPc} / \mathrm{Ag}(001)$ cluster, the MnPC structure was first re- 
laxed using the Vienna Ab inito Simulation Package (VASP, version 5.4.4 [93 96]), using the projector augmented-wave method, and applying the PBE exchange-correlation functional [97, 98]. The unit cell consists of one MnPc molecule and three Ag layers with a lateral size of $20.365 \times 20.365 \AA$. In accordance with Ref. [99], the manganese atom was adsorbed in the four-fold hollow position. During the structure optimization, only the $\mathrm{MnPc}$ atoms were allowed to relax, and the positions of the Ag atoms were kept frozen. Calculations were done at the $\Gamma$-point only, and dispersion interactions are accounted for by applying Grimme's dispersion correction with Becke-Jonson damping [100, 101]. The convergence criterion for the forces acting on each atom during the structure optimization was set to $0.01 \mathrm{eV} / \AA$ and for the self-consistent field algorithm to $2.7 \cdot 10^{-5} \mathrm{eV}$. The cutoff for the plane-wave basis set was set to $400 \mathrm{eV}$. The distance between the uppermost $\mathrm{Ag}$ layer of a unit cell and the lowermost Ag layer of the next unit cell in the $z$-direction is $12.2 \AA$, which ensures that during the structure optimization of $\mathrm{MnPc}$ the vacuum in $z$-direction is at least $9.0 \AA$.

For studying the hybridization function using a cluster approach, a pyramid-like cluster with 10 x 10 surface atoms and 4 layers containing $293 \mathrm{Ag}$ atoms was cut out from the optimized $\mathrm{MnPc} / \mathrm{Ag}(001)$ structure (Figure 5 b)). As with the $\mathrm{Co}(\mathrm{CO})_{4} / \mathrm{Cu}(001)$ system, a DFT single-point calculation was performed with Turbomole [88] (PBE/def2-SVP), and the hybridization functions were calculated as described in Section 3.

For the cluster calculations, the Fermi level $E_{F}$ for $\mathrm{Co}(\mathrm{CO})_{4} / \mathrm{Cu}(001)$ was set to the midpoint of the HOMO and LUMO energy. However, selecting this definition of $E_{F}$ was not possible in case of $\mathrm{MnPc} / \mathrm{Ag}(001)$, because we observed a negative HOMO-LUMO gap, which could not be solved using Fermi smearing during the SCF calculation. Thus, we decided for $\mathrm{MnPc} / \mathrm{Ag}(001)$ that $E_{F}=E_{\mathrm{HOMO}}$, as we have found for $\mathrm{Co}(\mathrm{CO})_{4} / \mathrm{Cu}(001)$ that this definition of $E_{F}$ in case of a negative HOMO-LUMO gap only barely affects the results (see supplementary information).

The def2-SVP basis has two sets of $d$ functions, one more tight one where each radial part is contracted from four primitive Gaussian functions, and one more diffuse one which consists of one primitive Gaussian. We define the correlated subsystem as the set of the five more 
tight (and more contracted) $d$ functions, as this gives good agreement with the hybridization functions obtained from periodic boundary conditions (see below).

\section{$5.2 \quad \mathrm{Co}(\mathrm{CO})_{4} / \mathrm{Cu}(001)$}

For the Kondo-relevant orbital $3 d_{\mathrm{x}^{2}-\mathrm{y}^{2}}$ in $\mathrm{Co}(\mathrm{CO})_{4} / \mathrm{Cu}(001), \operatorname{Im} \Delta_{i i}(\omega)$ was found to be increased in the range of $\omega=-3.0$ to $2.0 \mathrm{eV}$, with a significant enhancement of the hybridization at $\omega=-2.0 \mathrm{eV}$, as compared to an isolated $\mathrm{Co}$ atom on $\mathrm{Cu}(001)$ [35. Naively, one would expect the hybridization of the Co $3 d$ orbitals in the carbonyl species to be smaller than for an isolated $\mathrm{Co}$ on $\mathrm{Cu}(001)$, because of the increased Co-surface distance of $1.85 \AA$ in $\mathrm{Co}(\mathrm{CO})_{4} / \mathrm{Cu}(001)$ compared with $1.78 \AA$ in $\mathrm{Co} / \mathrm{Cu}(001)$.

However, a first estimate based on cutting away parts of the system suggested that this enhancement is indirectly (see Figure 4) caused by CO-surface interactions, i.e., by the ligands affecting how the surface interacts with the Co atom [35]. This was supported by an analysis of the density of states. In other words, the ligand-surface interaction appears to overcompensate the increased distance here, highlighting the importance of such interactions when one aims for controlling the Kondo effect chemically.

Given the potential change of the electronic structure by cutting away ligands, we will apply our local analysis to the hybridization function (introduced in Section 3) using a truncated cluster approach to this problem in the following. To this end, it is first analyzed whether such a cluster approach can qualitatively reproduce the Co hybridization function as obtained from a description under periodic boundary conditions. 
a)

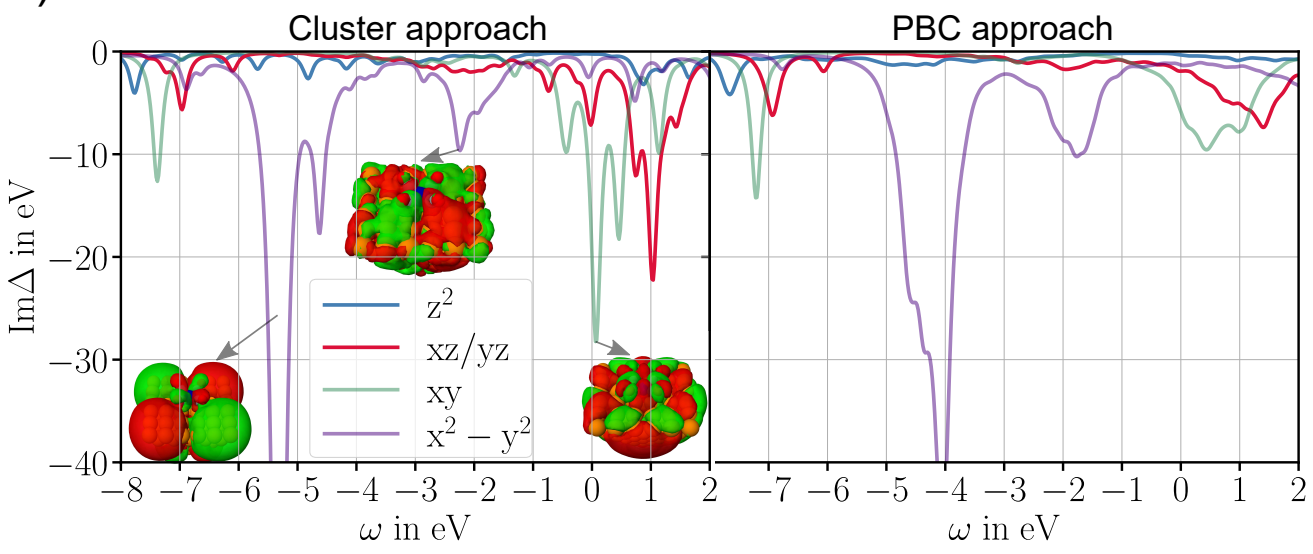

b) Co 3d local contributions to the hybridization functions

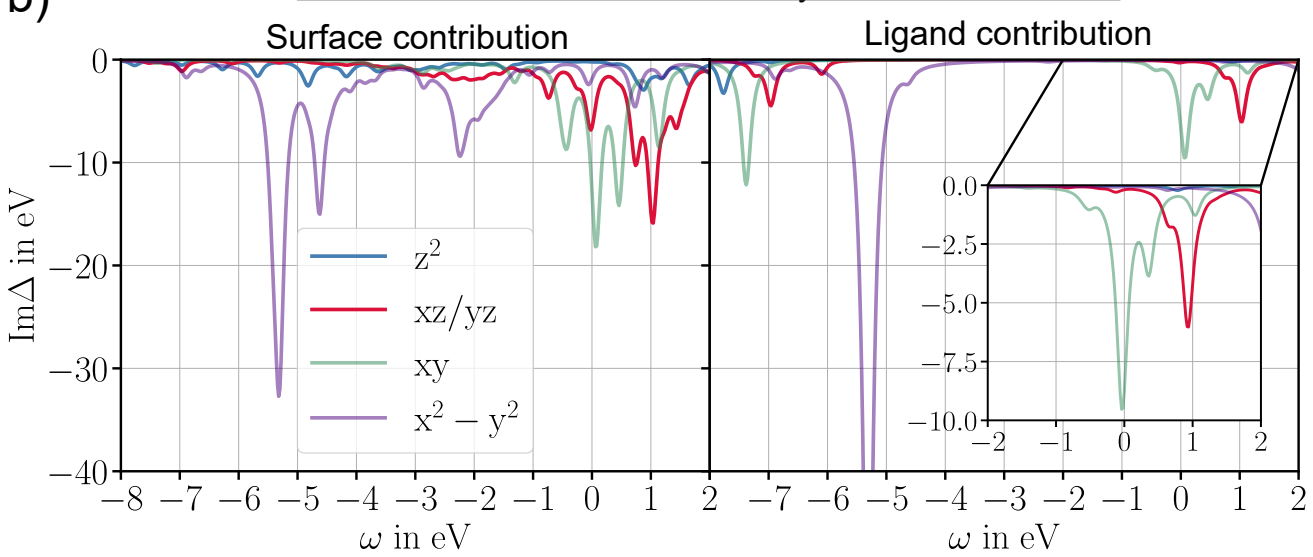

C $2 p$ hybridization functions from a cluster approach

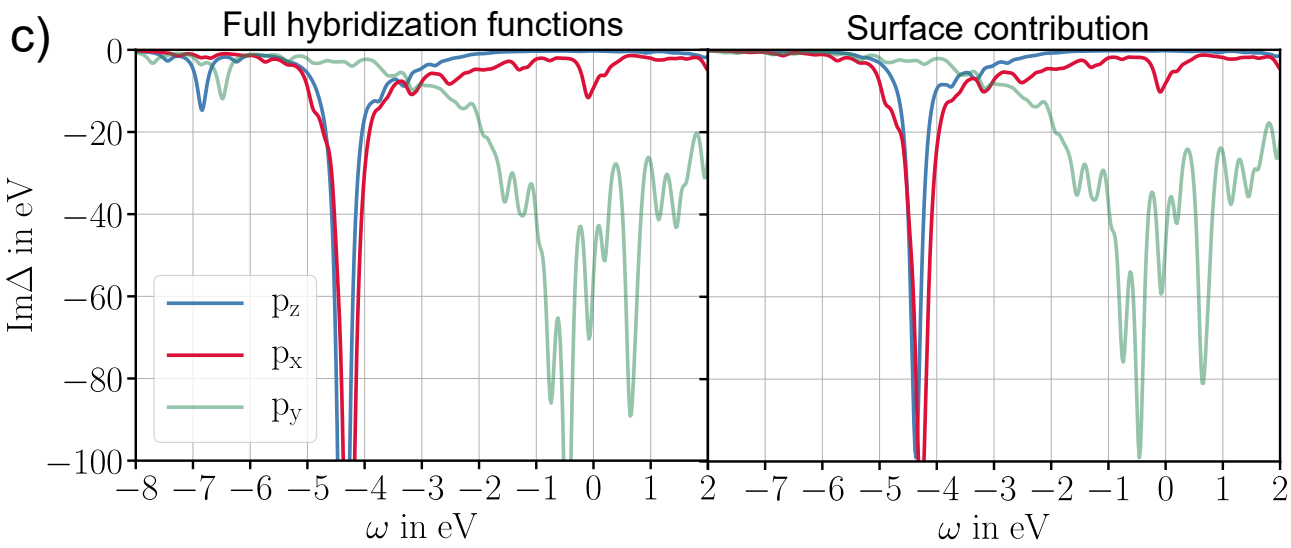

Figure 6: Imaginary parts of the Co $3 d$ hybridization function of $\mathrm{Co}(\mathrm{CO})_{4}$, relative to $E_{F}$ a) Left: $\operatorname{Im}\left(\Delta_{i i}\right)$ as obtained from a cluster approach. Bath orbitals are shown for selected features in the hybridization function (isosurface value 0.02). Right: $\operatorname{Im}\left(\Delta_{i i}\right)$ as obtained from a PBC approach (re-plotting of results obtained using PBE as the exchange-correlation functional in Ref. [35]). b) Surface (left) and ligand (right) contribution to $\operatorname{Im} \Delta_{i i}(\omega)$ of the Co $3 d$ orbitals in $\mathrm{Co}(\mathrm{CO})_{4} / \mathrm{Cu}(001)$ obtained from a cluster approach using Equation (8). c) Imaginary part of one of the $\mathrm{C} 2 p$ hybridization functions of $\mathrm{Co}(\mathrm{CO})_{4}$, as well as the surface contribution as calculated with Equation (8). For the cluster approach, $E_{F}$ was set to the mid-point of the HOMO-LUMO gap. 19 all plots (cluster and PBC), the smearing parameter (imaginary offset) $\eta$ was set to $0.1 \mathrm{eV}$. 


\subsubsection{Hybridization functions for $\mathrm{Co}(\mathrm{CO})_{4} / \mathrm{Cu}(001)$ from a cluster approach}

The imaginary part of the hybridization function of the Co $3 d$ orbitals of $\mathrm{Co}(\mathrm{CO})_{4} / \mathrm{Cu}(001)$ as obtained from a cluster approach is shown on the left-hand side of Figure 6 a). By comparing with the hybridization function from PBC shown on the right in the same figure, it becomes clear that the cluster approach can qualitatively reproduce the PBC hybridization function for the $\mathrm{Co} 3 d$ orbitals in $\mathrm{Co}(\mathrm{CO})_{4} / \mathrm{Cu}(001)$. For the Kondo-relevant orbital $3 d_{\mathrm{x}^{2}-\mathrm{y}^{2}}$, the features in the ranges of $\omega=-6.0 \mathrm{eV}$ to $-4.8 \mathrm{eV}$ and $\omega=-2.4 \mathrm{eV}$ to $-1.6 \mathrm{eV}$ are also present in the cluster approach. At the Fermi energy $(\omega=0.0 \mathrm{eV})$, the hybridization strength takes the following order for the truncated cluster, $\operatorname{Im} \Delta_{\mathrm{xy}}(0.0 \mathrm{eV})>\operatorname{Im} \Delta_{\mathrm{xz} / \mathrm{yz}}(0.0 \mathrm{eV})>$ $\operatorname{Im} \Delta_{\mathrm{x}^{2}-\mathrm{y}^{2}}(0.0 \mathrm{eV})>\operatorname{Im} \Delta_{\mathrm{z}^{2}}(0.0 \mathrm{eV})$, which qualitatively reproduces the results of the PBC approach.

As far as differences are concerned, in the cluster approach, the features are 1) less smooth than in the PBC approach (particularly above the Fermi energy), and 2) the large feature appears to be shifted towards lower energies. The first observation may be resulting from the metallic density of states not being fully reproduced by the truncated cluster. This less smooth behavior also correlates with the peaks going down to larger values. The second observation is not necessarily a shift, but is also consistent with a difference in intensities of the various sub-peaks making up the large feature. While this suggests caution when using the hybridization function from this truncated cluster for solving the AIM, the agreement appears sufficient for a local decomposition analysis.

\subsubsection{Local decomposition of the $3 d$ hybridization functions for $\mathrm{Co}(\mathrm{CO})_{4} / \mathrm{Cu}(001)$}

In the left-hand panel of Figure 6 b), the hybridization function is shown for the contributions from the surface to the total hybridization function, as obtained from Equation (8). The feature of the Kondo-relevant $d_{\mathrm{x}^{2}-\mathrm{y}^{2}}$ orbital in the range between $\omega=-2.5 \mathrm{eV}$ and $1.5 \mathrm{eV}$ comes purely from the $\mathrm{Cu}(001)$ surface, as can be seen by comparing with the ligand contribution (right-hand panel panel of Figure 6 b)). Most important for the Kondo physics 
is, however, the hybridization directly at the Fermi energy $(\omega=0.0 \mathrm{eV})$. The surface contribution to the hybridization function shows that $\operatorname{Im} \Delta_{\mathrm{x}^{2}-\mathrm{y}^{2}}$ is entirely dominated by the $\mathrm{Cu}$ surface in the area around the Fermi energy $(\omega=0.0 \mathrm{eV})$, and thus the small shift of the Fermi energy as compared to the PBC approach likely does not affect our conclusion here. In other words, there is no bath orbital with CO character that couples with the Co $3 d_{\mathrm{x}^{2}-\mathrm{y}^{2}}$ at the Fermi energy. This leads to the assumption that the CO ligands indirectly enhance the hybridization of the Kondo relevant orbital at the Fermi energy, due to CO-surface interactions modifying the substrate electronic structure, resulting in the rather large Kondo temperature found in scanning tunneling spectroscopy experiments [35, 85, 86].

That the ligand-surface interactions play an important role here is supported by the strong hybridization of the C $2 p$ orbitals with the surface, as depicted in Figure 6 c). In contrast to the $d_{\mathrm{x}^{2}-\mathrm{y}^{2}}$ orbital, at the Fermi energy the $d_{\mathrm{xy}}$ orbital hybridizes the strongest of all Co $3 d$ orbitals in the tetracarbonyl system. Within our approach, we can identify that this comes from a bath orbital with $\pi^{*}$-character on the CO ligands (see Figure 6 a)), which is energetically close to the Fermi energy. On the other hand, the bath orbital with $\mathrm{CO} \sigma$ character (at $\omega=-5.4 \mathrm{eV}$ ), which could hybridize with the $d_{\mathrm{x}^{2}-\mathrm{y}^{2}}$ orbital, is at an energetic position far away from the Fermi energy (while the bath orbital with $\sigma^{*}$-character lies above $\omega>2.0 \mathrm{eV}$, which is beyond the energy range shown in Figure 6 a)). This observation is in particular interesting for designing Kondo systems, as it is known from the simplest Anderson impurity model that a too strong hybridization can lead to the destruction of the Kondo resonance (i.e. if the hybridization is much larger than the Coulomb interaction on the impurity, one is in the so-called non-interacting regime of the Anderson impurity model [33]).

\section{$5.3 \mathrm{MnPc} / \operatorname{Ag}(001)$}

It was shown that in the case of $\mathrm{Co}(\mathrm{CO})_{4} / \mathrm{Cu}(001)$, the interaction of the ligand with the substrate can give rise to an increased hybridization at the Fermi energy, thus resulting in an increased Kondo temperature. While a certain hybridization is required for the Kondo 
effect, a too strong hybridization at the Fermi energy can suppress it [87, 102]. In this context, $\mathrm{MnPc} / \mathrm{Ag}(001)$ is an interesting candidate for applying our approach to decompose the hybridization function, because it has been suggested that for two of its $3 d$ orbitals $\left(3 d_{\mathrm{xz} / \mathrm{yz}}\right)$, such a large hybridization function indeed keeps them from being Kondo active [87]. While as a high-spin $\mathrm{Mn}$ (II) complex it has formally five unpaired electrons, which could all potentially exhibit Kondo screening, two other orbitals do not hybridize close to the Fermi energy $\left(3 d_{\mathrm{xy}}\right.$ and $\left.3 d_{\mathrm{x}^{2}-\mathrm{y}^{2}}\right)$, so that only the Mn $3 d_{\mathrm{z}^{2}}$ is likely to be Kondo-screened [87]. As an additional interesting feature (not to be explored further in this work), this Kondo screening and thus the Kondo temperature can be controlled by dehydrogenation [34].
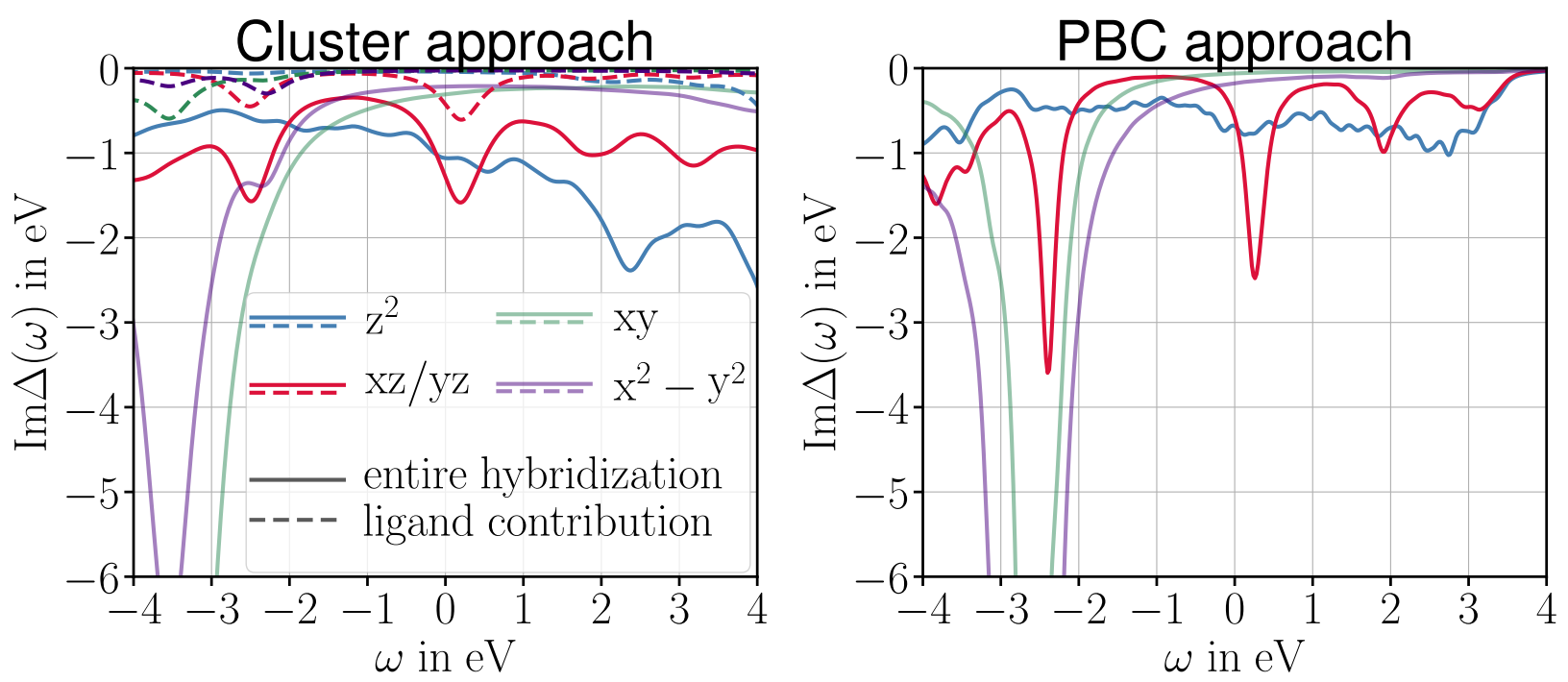

Figure 7: Left: Imaginary part of the Mn $3 d$ hybridization function, relative to $E_{F}$, in $\mathrm{MnPc} / \mathrm{Ag}(001)$ as obtained from $\mathrm{PBE} /$ def2-SVP using a truncated cluster approach, applying a smearing (imaginary offset) of $0.3 \mathrm{eV}$. Dashed lines shows the ligand contribution to the entire hybridization function (according to Equation (8p). Right: Imaginary part of the Mn $3 d$ hybridization functions for $\mathrm{MnPc} / \mathrm{Ag}(001)$ using periodic boundary conditions, applying a smearing (imaginary offset) of $0.1 \mathrm{eV}$. The larger smearing in the cluster approach was chosen here to make the plots as similar as possible. For the cluster calculation, $E_{F}$ was set to the energy of the HOMO.

\subsubsection{Hybridization functions for $\mathrm{MnPc} / \mathrm{Ag}(001)$ from a cluster approach}

The Mn $3 d$ hybridization function obtained from a truncated cluster approach (left-hand side of Figure 7) recovers all essential features as obtained from a PBC approach (right-hand 
side of Figure 7; also compare Ref. [34]). It assumes in general larger values as compared to the PBC approach, even though we applied a larger value for the effective smearing in the cluster case. This could explain why in our case the hybridization functions of the $3 d_{\mathrm{xy}}$ and $3 d_{\mathrm{x}^{2}-\mathrm{y}^{2}}$ orbitals around the Fermi energy $(\omega=0 \mathrm{eV})$ are larger than reported in Refs. [87] and [34]. Nevertheless, we can confirm that these orbitals are significantly less hybridized at the Fermi energy than the $3 d_{\mathrm{z}^{2}}$ and $3 d_{\mathrm{xz} / \mathrm{yz}}$ orbitals.

\subsubsection{Local decomposition of the $3 d$ hybridization functions for $\mathrm{MnPc} / \mathrm{Ag}(001)$}

For the Kondo-relevant $3 d_{\mathrm{z}^{2}}$ orbital, we observe a flat hybridization function around the Fermi energy, with no direct contribution from the Pc ligand over the entire energy range shown here (solid and dotted blue lines in left-hand side of Figure 7). The hybridization function at $E_{F}$ of this orbital is significantly larger than for an isolated $\mathrm{Mn}$ on $\mathrm{Ag}(001)$ (see supplementary information), and our local decomposition thus suggests that this enhancement is indirectly caused by ligand-surface interactions, similar to the Kondo-active orbital in $\mathrm{Co}(\mathrm{CO})_{4} / \mathrm{Cu}(001)$.

After having focused on the Kondo-active orbitals so far, we will now discuss an example of orbitals which could be Kondo-active due to being half-filled, but which are not due to their too large hybridization function at $E_{F}$ : For the $3 d_{\mathrm{xz} / \mathrm{yz}}$ orbitals, it was argued previously based on the relatively sharp peak at the Fermi energy that these orbitals couple dominantly to the Pc ligand with its discrete levels rather than to the surface [87]. Our local decomposition allows us to test this hypothesis. Indeed, the Pc ligand contribution (dashed red line in left-hand side of Figure 7] to the total hybridization for these orbitals is around $40 \%$, indicating an enhancement of the $3 d_{\mathrm{xz} / \mathrm{yz}}$ hybridization due to direct interactions with the Pc ligand. The remaining 60\% originate from the substrate (also see supplementary material), where interestingly, the peak at the Fermi energy would still be present after subtraction of the ligand contribution. The $60 \%$ surface contribution of these orbitals is already larger than the total hybridization function of the same orbitals in an isolated Mn on $\operatorname{Ag}(001)$ (see Figure 5 of the supplementary information), from which we can conclude 
that the hybridization function at $E_{F}$ is also indirectly enhanced through ligand-surface interactions.

Altogether, while for the Kondo-active orbitals of $\mathrm{Co}(\mathrm{CO})_{4} / \mathrm{Cu}(001)$ and $\mathrm{MnPc} / \mathrm{Ag}(001)$, the ligand-induced enhancement of the hybridization at the Fermi energy is a purely indirect effect of the ligand modifying the surface electronic structure, we have both such an indirect and a direct effect of the ligand on the two potentially Kondo-active $3 d_{\mathrm{xz} / \mathrm{yz}}$ orbitals in $\mathrm{MnPc} / \operatorname{Ag}(001)$. This could explain why the hybridization function is so strong for them that their Kondo activity is suppressed.

\section{Conclusion}

Truncated-cluster approaches are useful for describing substrate-adsorbate systems, as they allow for using a variety of exchange-correlation functionals (see supplementary material for a discussion), and for straightforward interpretations in terms of molecular orbitals. In this work, we have shown that the hybridization functions from such a truncated-cluster approach can qualitatively reproduce hybridization functions obtained under periodic boundary conditions. In follow-up studies, it would be interesting to systematically check the optimal size and shape of a cluster for this purpose, and the hybridization function could serve as a simple yet encompassing diagnostic tool to assess the suitability of clusters to model extended surfaces for electronic properties.

We introduced a local decomposition of the hybridization function, which allows for quantifying the contributions from different parts of the environment, such as ligands or surface atoms, thus allowing for a qualitative understanding of the Kondo effect. This is important for chemical control of strong electron correlation [99, 103, 104]. Based on this decomposition, we could show that the enhancement of the hybridization function at the Fermi energy for the Kondo-active orbital in $\mathrm{Co}(\mathrm{CO})_{4} / \mathrm{Cu}(001)$ compared with a pure $\mathrm{Cu}$ atom on the same surface can be purely attributed to the surface; this enhancement thus results from an indirect effect of the ligands. By contrast, in $\mathrm{MnPc} / \mathrm{Ag}(001)$, we have both such indirect 
and direct contributions from the ligands for two potentially Kondo-active orbitals, which makes the hybridization function so large that their Kondo activity is suppressed (leaving the Kondo activity to only one orbital).

It would also be worthwhile to combine our local decomposition scheme with two recently suggested approaches, (1) an improved DFT-based projection-operator diabatization method, which combines orthogonality with localization also for larger basis sets, and which is particularly well suited for molecules on surfaces [62], and (2) an approach towards a minimal yet accurate tight-binding basis from sub-diagonalization of the Hamiltonian [105]. Also, combinations with approaches capable of describing the Kondo effect in organic radicals [50] would be desirable, or with new electronic structure methods in which a correlated subsystem is embedded in a molecular environment [106], and it would be interesting to ask how information on local contributions to hybridization functions relates to (local) measures

of correlation [107-109] Given the equivalence between the energy-dependent hybridization function and the self-energy describing electronic coupling of a system to its environment, for example a molecular wire linking to two electrodes, our local decomposition scheme could also be helpful to elucidate which parts of this environment have the largest effect on the molecule. This could help elucidate structure-property relationships in molecular electronics and spintronics.

\section{Supplementary Material}

As supplementary material, we provide a detailed discussion on the effects of the negative HOMO-LUMO gap in our clusters and of the choice of approximate exchange-correlation functional on the hybridization function. Furthermore we discuss the contributions of the surface and the basis functions on the $3 d$ metal atom which do not make up the correlated subsystem in the MnPc system, the hybridization function of an atomic Mn adsorbate on $\operatorname{Ag}(001)$, and the implementation of an alternative way of evaluating the full hybridization function in HyDRA. In addition, we provide Cartesian coordinates for our clusters 
and VASP input files for our calculations under periodic boundary conditions. A working version of our code HYDRA for review can be found here: https://attachment.rrz.unihamburg.de/6ee20bed/si.tgz We are currently finalizing the code for public sharing.

\section{Acknowledgments}

We would like to thank HLRN and the high-performance computing centre at University of Hamburg for computational resources. This work is supported by Deutsche Forschungsgemeinschaft (DFG) via project HE 5675/6-1. We thank Martin Sebastian Zöllner for providing us with a Fortran code for extracting Fock and overlap matrices from TuRBOmole output.

\section{Data Availability}

The data that supports the findings of this study are available within the article and its supplementary material.

\section{Dedication}

We would like to dedicate this article to Myriam Sarachik, who gave the first experimental confirmation of the Kondo effect, and to Sigrid Peyerimhoff, who has always been an inspiration through her pioneering work on correlated electronic structure and beyond.

\section{References}

[1] G. D. Scott and D. Natelson, ACS Nano 4, 3560 (2010). 
[2] Y. hui Zhang, S. Kahle, T. Herden, C. Stroh, M. Mayor, U. Schlickum, M. Ternes, P. Wahl, and K. Kern, Nat Commun 4 (2013), 10.1038/ncomms3110.

[3] D. Jacob, J. Phys.: Condens. Matter 30, 354003 (2018).

[4] C. Romeike, M. R. Wegewijs, W. Hofstetter, and H. Schoeller, Phys. Rev. Lett. 97, 206601 (2006).

[5] R. Frisenda, R. Gaudenzi, C. Franco, M. Mas-Torrent, C. Rovira, J. Veciana, I. Alcon, S. T. Bromley, E. Burzuri, and H. S. J. van der Zant, Nano Lett. 15, 3109 (2015).

[6] W. Liang, M. P. Shores, M. Bockrath, J. R. Long, and H. Park, Nature 417, 725 (2002).

[7] J. Liu, H. Isshiki, K. Katoh, T. Morita, K. B. Brian, M. Yamashita, and T. Komeda, J. Am. Chem. Soc. 135, 651 (2013).

[8] R. Requist, P. P. Baruselli, A. Smogunov, M. Fabrizio, S. Modesti, and E. Tosatti, Nature Nanotech. 11, 499 (2016).

[9] L. Gao, W. Ji, Y. B. Hu, Z. H. Cheng, Z. T. Deng, Q. Liu, N. Jiang, X. Lin, W. Guo, S. X. Du, and W. A. Hofer, Phys. Rev. Lett. 99, 106402 (2007).

[10] Z. Huang, Y. Zhang, Y. He, H. Song, C. Yin, and K. Wu, Chem. Soc. Rev. 46, 1955 $(2017)$.

[11] T. Pope, S. Du, H.-J. Gao, and W. A. Hofer, Chem. Commun. 54, 5508 (2018).

[12] L. Kouvenhoven and L. Glazman, Physics World 14, 33 (2001).

[13] S. M. Cronenwett, T. H. Oosterkamp, and L. P. Kouwenhoven, Science 281, 540 (1998), http://science.sciencemag.org/content/281/5376/540.full.pdf .

[14] M. Ternes, Prog. Surf. Sci. 92, 83 (2017).

[15] T. Choi, S. Bedwani, A. Rochefort, C.-Y. Chen, A. J. Epstein, and J. A. Gupta, Nano Lett. 10, 4175 (2010). 
[16] T. Choi, M. Badal, S. Loth, J.-W. Yoo, C. P. Lutz, A. J. Heinrich, A. J. Epstein, D. G. Stroud, and J. A. Gupta, Nano Lett. 14, 1196 (2014).

[17] M.-L. Bocquet, N. Lorente, R. Berndt, and M. Gruber, Angew. Chem. Int. Ed. 58, $821(2019)$.

[18] A. Zhao, Q. Li, L. Chen, H. Xiang, W. Wang, S. Pan, B. Wang, X. Xiao, J. Yang, J. G. Hou, and Q. Zhu, Science 309, 1542 (2005).

[19] R. Temirov, A. Lassise, F. Anders, and F. Tautz, Nanotechnology 19, 065401 (2008).

[20] N. Tsukahara, E. Minamitani, Y. Kim, M. Kawai, and N. Takagi, J. Chem. Phys. 141, 054702 (2014).

[21] T. Knaak, M. Gruber, C. Lindström, M.-L. Bocquet, J. Heck, and R. Berndt, Nano Lett. 17, 7146 (2017).

[22] P. Stoll, C. Lotze, J. N. Ladenthin, T. R. Umbach, I. Fernández-Torrente, and K. J. Franke, J. Phys.: Condens. Matter 30, 454002 (2018).

[23] P. Jacobson, T. Herden, M. Muenks, G. Laskin, O. Brovko, V. Stepanyuk, M. Ternes, and K. Kern, Nature Commun. 6, 8536 (2015).

[24] V. Iancu, A. Deshpande, and S.-W. Hla, Nano Lett. 6, 820 (2006).

[25] J. Meyer, R. Ohmann, A. Nickel, C. Toher, R. Gresser, K. Leo, D. A. Ryndyk, F. Moresco, and G. Cuniberti, Phys. Rev. B 93, 155118 (2016).

[26] J. Parks, A. Champagne, T. Costi, W. Shum, A. Pasupathy, E. Neuscamman, S. FloresTorres, P. Cornaglia, A. Aligia, C. Balseiro, et al., Science 328, 1370 (2010).

[27] B. Maughan, P. Zahl, P. Sutter, and O. L. A. Monti, J. Phys. Chem. Lett. 8, 1837 $(2017)$.

[28] J. Bouaziz, F. S. M. Guimarães, and S. Lounis, Nat. Commun. 11, 6112 (2020). 
[29] A. Valli, M. P. Bahlke, A. Kowalski, M. Karolak, C. Herrmann, and G. Sangiovanni, Phys. Rev. Research 2, 033432 (2020).

[30] F. Liu, C. Duan, and H. J. Kulik, J. Chem. Phys. Lett. 11, 8067 (2020),

[31] C. R. Jacob and M. Reiher, Int. J. Quantum Chem. 112, 3661 (2012).

[32] J. Brüggemann and C. R. Jacob, Faraday Discuss. 224, 56 (2020).

[33] A. C. Hewson, The Kondo Problem to Heavy Fermions (Cambridge University Press, Cambridge, 1997).

[34] J. Kügel, M. Karolak, A. Krönlein, J. Senkpiel, P.-J. Hsu, G. Sangiovanni, and M. Bode, Physical Review B 91, 235130 (2015).

[35] M. P. Bahlke, P. Wahl, L. Diekhöner, and C. Herrmann, Journal of Applied Physics 125, 142910 (2019).

[36] P.-O. Löwdin, J. Chem. Phys. 18, 365 (1950).

[37] F. Heidar-Zadeh, P. W. Ayers, T. Verstraelen, I. Vinogradov, E. Vöhringer-Martinez, and P. Bultinck, J. Phys. Chem. A 122, 4219 (2018).

[38] M. G. Reuter and R. J. Harrison, J. Chem. Phys. 139, 114104 (2013).

[39] M. Soriano and J. J. Palacios, Phys. Rev. B 90, 075128 (2014).

[40] A. H. Mühlbach and M. Reiher, J. Chem. Phys. 149, 184104 (2018).

[41] R. F. W. Bader, Atoms in molecules: a quantum theory, Bader (Oxford University Press, New York, 1994).

[42] A. E. Clark and E. R. Davidson, Int. J. Quantum Chem. 93, 384 (2003).

[43] A. E. Reed, R. B. Weinstock, and F. Weinhold, J. Chem. Phys. 83, 735 (1984).

[44] E. R. Davidson, J. Chem. Phys. 46, 3320 (1967). 
[45] I. Mayer, Chem. Phys. Lett. 424, 499 (1995).

[46] F. Hirshfeld, Theor. Chim. Acta 44, 129 (1977).

[47] R. S. Mulliken, J. Chem. Phys. 23, 1833 (1955).

[48] B. Surer, M. Troyer, P. Werner, T. O. Wehling, A. M. Luchli, A. Wilhelm, and A. I. Lichtenstein, Phys. Rev. B 85 (2012), 10.1103/physrevb.85.085114.

[49] E. Gorelov, T. O. Wehling, A. N. Rubtsov, M. I. Katsnelson, and A. I. Lichtenstein, Phys. Rev. B 80, 155132 (2009).

[50] A. Droghetti and I. Rungger, Phys. Rev. B 95, 085131 (2017).

[51] W. H. Appelt, A. Droghetti, L. Chioncel, M. M. Radonjić, E. Muñoz, S. Kirchner, D. Vollhardt, and I. Rungger, Nanoscale 10, 17738 (2018).

[52] J. C. Slater, Quantum Theory of Atomic Structure (McGraw-Hill, New York, 1960).

[53] E. Gull, A. J. Millis, A. I. Lichtenstein, A. N. Rubtsov, M. Troyer, and P. Werner, Rev. Mod. Phys. 83, 349 (2011).

[54] Y. Lu and M. W. Haverkort, The European Physical Journal Special Topics 226, 2549 (2017).

[55] H. Shinaoka, E. Gull, and P. Werner, Comput. Phys. Commun. 215, 128 (2017).

[56] D. Jacob, J. Phys.: Condens. Matter 27, 245606 (2015).

[57] A. Nitzan, ed., Chemical Dynamics in Condensed Phases: Relaxation, Transfer, and Reactions in C (Oxford University Press, Oxford, 2006).

[58] P.-O. Löwdin, Adv. Quantum. Chem. 5, 185 (1970).

[59] I. Mayer, Int. J. Quantum Chem. 90, 63 (2002).

[60] I. Kondov, M. Č́́žek, C. Benesch, H. Wang, and M. Thoss, J. Phys. Chem. C 111, 11970 (2007). 
[61] Z. Futera and J. Blumberger, J. Phys. Chem. C 121, 19677 (2017).

[62] S. Ghan, C. Kunkel, K. Reuter, and H. Oberhofer, J. Chem. Theory Comput. 16, $7431(2020)$.

[63] X. Gonze, F. Jollet, F. Abreu Araujo, D. Adams, B. Amadon, T. Applencourt, C. Audouze, J.-M. Beuken, J. Bieder, A. Bokhanchuk, E. Bousquet, F. Bruneval, D. Caliste, M. Cote, F. Dahm, F. Da Pieve, M. Delaveau, M. Di Gennaro, B. Dorado, C. Espejo, G. Geneste, L. Genovese, A. Gerossier, M. Giantomassi, Y. Gillet, D. Hamann, L. He, G. Jomard, J. Laflamme Janssen, S. Le Roux, A. Levitt, A. Lherbier, F. Liu, I. Lukacevic, A. Martin, C. Martins, M. Oliveira, S. Ponce, Y. Pouillon, T. Rangel, G.M. Rignanese, A. Romero, B. Rousseau, O. Rubel, A. Shukri, M. Stankovski, M. Torrent, M. Van Setten, B. Van Troeye, M. Verstraete, D. Waroquiers, J. Wiktor, B. Xu, A. Zhou, and J. Zwanziger, Comput. Phys. Commun. 205, 106 (2016).

[64] X. Gonze, B. Amadon, G. Antonius, F. Arnardi, L. Baguet, J.-M. Beuken, J. Bieder, F. Bottin, J. Bouchet, E. Bousquet, N. Brouwer, F. Bruneval, G. Brunin, T. Cavignac, J.-B. Charraud, W. Chen, M. Cote, S. Cottenier, J. Denier, G. Geneste, P. Ghosez, M. Giantomassi, Y. Gillet, O. Gingras, D. R. Hamann, G. Hautier, X. He, N. Helbig, N. Holzwarth, Y. Jia, F. Jollet, W. Lafargue-Dit-Hauret, K. Lejaeghere, M. A. Marques, A. Martin, C. Martins, H. P. Miranda, F. Naccarato, K. Persson, G. Petretto, V. Planes, Y. Pouillon, S. Prokhorenko, F. Ricci, G.-M. Rignanese, A. H. Romero, M. M. Schmitt, M. Torrent, M. J. van Setten, B. Van Troeye, M. J. Verstraete, G. Zerah, and J. W. Zwanziger, Comput. Phys. Commun. 248, 107042 (2020).

[65] H. Shinaoka, S. Hoshino, M. Troyer, and P. Werner, Phys. Rev. Lett. 115, 156401 $(2015)$.

[66] T. Jabben, N. Grewe, and S. Schmitt, Phys. Rev. B 85, 045133 (2012).

[67] G. Guennebaud, B. Jacob, et al., "Eigen v3," http://eigen.tuxfamily.org (2010).

[68] OpenMP Application Programming Interface Version 5.0, https://www.openmp.org/specifications. 
[69] "Mpi-3.1, https://www.mpi-forum.org/docs/mpi-3.1/mpi31-report.pdf," .

[70] M. Head-Gordon and J. C. Tully, Phys. Rev. B 46, 1853 (1992).

[71] R. van Santen, J. Mol. Catal. A: Chem. 115, 405 (1997).

[72] W. B. Schneider, U. Benedikt, and A. A. Auer, ChemPhysChem 14, 2984 (2013).

[73] O. Tsendra, A. M. Scott, L. Gorb, A. D. Boese, F. C. Hill, M. M. Ilchenko, D. Leszczynska, and J. Leszczynski, J. Phys. Chem. C 118, 3023 (2014).

[74] R. J. Maurer, M. Askerka, V. S. Batista, and J. C. Tully, Phys. Rev. B 94, 115432 (2016).

[75] L. Groß, M. P. Bahlke, T. Steenbock, C. Klinke, and C. Herrmann, J. Comput. Chem. 38, 861 (2017).

[76] J. Banerjee, S. Behnle, M. C. E. Galbraith, V. Settels, B. Engels, R. Tonner, and R. F. Fink, J. Comput. Chem. 39, 844 (2018).

[77] S. N. Yaliraki, A. E. Roitberg, C. Gonzalez, V. Mujica, and M. A. Ratner, J. Chem. Phys. 111, 6997 (1999),

[78] Y. Luo, C.-K. Wang, and Y. Fu, J. Chem. Phys. 117, 10283 (2002).

[79] A. Nitzan and M. A. Ratner, Science 300, 1384 (2003).

[80] C. Herrmann, G. C. Solomon, and M. A. Ratner, J. Am. Chem. Soc. 132, 3682 (2010).

[81] R. Hayakawa, M. A. Karimi, J. Wolf, T. Huhn, M. S. Zöllner, C. Herrmann, and E. Scheer, Nano Lett. 16, 4960 (2016).

[82] M. Strange, I. S. Kristensen, K. S. Thygesen, and K. W. Jacobsen, J. Chem. Phys. 128, 114714 (2008).

[83] K. Thygesen and K. Jacobsen, Chem. Phys. 319, 111 (2005). 
[84] G. Knizia, J. $\quad$ Chem. Theory $\quad$ Comput. 9, 4834 (2013), https://doi.org/10.1021/ct400687b.

[85] P. Wahl, L. Diekhöner, M. A. Schneider, L. Vitali, G. Wittich, and K. Kern, Phys. Rev. Lett. 93, 176603 (2004).

[86] P. Wahl, L. Diekhöner, G. Wittich, L. Vitali, M. A. Schneider, and K. Kern, Phys. Rev. Lett. 95, 166601 (2005).

[87] J. Kügel, M. Karolak, J. Senkpiel, P.-J. Hsu, G. Sangiovanni, and M. Bode, Nano letters 14, 3895 (2014).

[88] "TURBOMOLE V7.1 2016, a development of University of Karlsruhe and Forschungszentrum Karlsruhe GmbH, 1989-2007, TURBOMOLE GmbH, since 2007; available from http://www .turbomole. com." .

[89] J. P. Perdew and Y. Wang, Phys. Rev. B 45, 13244 (1992).

[90] J. P. Perdew, K. Burke, and M. Ernzerhof, Phys. Rev. Lett. 77, 3865 (1996).

[91] F. Weigend and R. Ahlrichs, Phys. Chem. Chem. Phys. 7, 3297 (2005).

[92] A. Hellweg, C. Hättig, S. Höfener, and W. Klopper, Theor. Chem. Acc. 117, 587 (2007).

[93] G. Kresse and J. Hafner, Phys. Rev. B 49, 14251 (1994).

[94] G. Kresse, Journal of Non-Crystalline Solids 192-193, 222 (1995), structure of NonCrystalline Materials 6.

[95] G. Kresse and J. Furthmüller, Phys. Rev. B 54, 11169 (1996).

[96] G. Kresse and J. Furthmüller, Computational Materials Science 6, 15 (1996)

[97] P. E. Blöchl, Phys. Rev. B 50, 17953 (1994). 
[98] G. Kresse and D. Joubert, Phys. Rev. B 59, 1758 (1999).

[99] J. Kügel, M. Karolak, A. Krönlein, J. Senkpiel, G. Sangiovanni, and M. Bode, Phys. Rev. B , 235130 (2015).

[100] S. Grimme, J. Antony, S. Ehrlich, and H. Krieg, J. Chem. Phys. 132, 154104 (2010).

[101] S. Grimme, S. Ehrlich, and L. Goerigk, J. Comput. Chem. 32, 1456 (2011).

[102] D. Jacob, M. Soriano, and J. Palacios, Physical Review B 88, 134417 (2013).

[103] J. Kügel, M. Karolak, A. Krönlein, D. Serrate, M. Bode, and G. Sangiovanni, npj quantum mater 3, 53 (2018), 1811.11294v1.

[104] Y. Wang, X. Li, and J. Yang, J. Chem. Phys. 151, 224704 (2019).

[105] G. Gandus, A. Valli, D. Passerone, and R. Stadler, J. Chem. Phys. 153, 194103 (2020).

[106] T. N. Lan, A. A. Kananenka, and D. Zgid, J. Chem. Theory Comput. 12, 4856 (2016).

[107] C. Stemmle and B. Paulus, Int. J. Quantum Chem. 119, e26007.

[108] C. J. Stein and M. Reiher, Mol. Phys. (2017), dOI: 10.1080/00268976.2017.1288934.

[109] S. Grimme and A. Hansen, Angew. Chem. Int. Ed. 54, 12308 (2015), https://onlinelibrary.wiley.com/doi/pdf/10.1002/anie.201501887. 